\title{
Estudio comparativo en pacientes en hemodiafiltración on-line postdilucional de alta eficacia con diferentes flujos de líquido dializante con tiempo programado y tiempo real
}

\author{
Sonia Aznar Barbero, Ramón Bel Cegarra, María Magdalena Badallo Mira, David Pagán Escribano, Asensio \\ Bartolomé García Nicolás
}

\section{Hospital Santa Lucía de Cartagena. Murcia}

\section{Introducción:}

La dosis de diálisis (DD) es un elemento fundamental en la diálisis adecuada ${ }^{1,2}$ y en la supervivencia del paciente $\mathrm{e}^{3,4,5}$. La Hemodiafiltración on-line logra DD mayores y altos volúmenes de reinfusión, por tanto el aumento en volumen convectivo conlleva un descenso en la mortali$\operatorname{dad}^{13,14}$.

En la DD influyen 4 elementos: tiempo, flujo sanguíneo $(Q b)$, coeficiente de transferencia de masa de urea (KoA) y flujo del baño (Qd)15. Algunos autores ${ }^{16,17}$ señalan que aumentando Qd se obtiene entre $5-10 \%{ }^{18}$ de mejora en la $\mathrm{DD}$, pero este aumento incrementa el coste.

El objetivo es evaluar si con Qd $800 \mathrm{ml} / \mathrm{min}$ y tiempo programado 240 minutos conseguimos mayor eficacia que con Qd $500 \mathrm{ml} / \mathrm{min}$ y tiempo programado 240 minutos y con Qd 500 l/min y tiempo efectivo 240 minutos.

\section{Material y métodos:}

Estudio prospectivo sobre población prevalente en hemodiálisis, con criterio de inclusión pacientes mayores de edad en hemodiafiltración on-line postdilucional. En cada paciente se realizan 18 sesiones consecutivas variando Qd y tiempo con siguiente esquema:

- Fase 1: 6 sesiones con Qd $800 \mathrm{ml} / \mathrm{min}$ y tiempo programado 240 minutos (F1).

- Fase 2: 6 sesiones con Qd $500 \mathrm{ml} / \mathrm{min}$ y tiempo programado 240 minutos (F2).

- Fase 3: 6 sesiones con Qd $500 \mathrm{ml} / \mathrm{min}$ y tiempo efectivo 240 minutos (F3).
Se mantiene $\mathrm{Qb}$ en $400 \mathrm{ml} / \mathrm{min}$ de flujo programado. Los dializadores son membranas sintéticas de alta permeabilidad de $2,1 \mathrm{~m}^{2}$.

El Volumen total de reinfusión (VTR) se mide de forma automatizada y la DD por dialisancia iónica (Kt ajustado a superficie corporal ASC).

EI análisis estadístico se realiza mediante SPSS 13.0 para Windows.

El contraste de hipótesis para variables cuantitativas mediante t-student y ANOVA, y chi-cuadrado para cualitativas. Significación estadística $p<0,05$.

\section{Resultados:}

Analizamos 432 sesiones [24 pacientes, 50\% hombres, de $58,5 \pm 17,6$ años. Las etiologías más prevalentes eran glomerular $(33,3 \%)$, intersticial $(16,7 \%)$ y vascular $(16,7 \%)$. El tiempo de permanencia en IRT $45,3 \pm 49,4$ meses, $91,7 \%$ portadores de Fistula Arteriovenosa].

$\mathrm{Kt}>$ en $\mathrm{F} 3(56,5 \pm 3,8$ litros, $\mathrm{p}<0,05)$ con respecto a $\mathrm{Fl}$ $(53,6 \pm 3,6$ litros $)$ y $F 2(53,7 \pm 3,9$ litros $)$. No encontramos diferencias significativas en $\mathrm{F} 1$ vs $\mathrm{F} 2$.

VTR > en $F 3(26,3 \pm 3,8$ litros $p<0,05)$ con respecto a

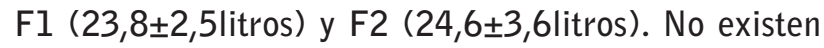
diferencias significativas en VTR entre F1 y F2.

No existen diferencias significativas en porcentaje de reducción de urea (PRU) entre $\mathrm{Fl}(79,3 \pm 6,5 \%)$, F2 $(79,2 \pm 6,6 \%)$ y F3 $(79,08 \pm 5,4 \%)$. 
91,7\% de los pacientes alcanzan Kt óptimo ASC en F1 y F2, siendo el $95,83 \%$ en F3.

Para lograr tiempo efectivo de 240 minutos se sumó 10 minutos al tiempo programado.

\section{Discusión y conclusiones:}

No existen diferencias en la eficacia dialítica entre Qd $500 \mathrm{ml} / \mathrm{min}$ y Qd $800 \mathrm{ml} / \mathrm{min}$, mientras que con el aumento de 10 minutos de media en cada sesión, incrementamos la dosis de diálisis en casi 3 litros y el VTR en un $9 \%$, reduciendo a la mitad el número de pacientes que no logran los 20 litros de volumen de reinfusión. $Y$ esto, sin coste adicional especialmente por las diferencias de envasado del bicarbonato.

Esta opción se podrá tener en cuenta en el elenco terapéutico para la consecución de objetivos individuales en aras de optimizar recursos.

\section{Referencias Bibliográficas}

1. Held PJ, Port FK, Wolfe RA, Stannard DC, Carrol $C E$, Dagirdas JT, et al. The dose of hemodialysis and patients mortality. Kidney Int1996; 50:550-6.

2. Hakim RM, Breyer J, Ismail N, Schulman G. Effects of dose of dialysis on morbidity and mortality. Am J Kidney Dis 1994; 23:661-9.

3. Maduell F. Dosis de hemodiálisis: condición sine qua non de diálisis adecuada. Nefrología 1999; 19 (Supl 4):51-3.

4. Maduell F, García M, Alcázar R. Dosificación y adecuación del tratamiento dialítico. Guías SEN. Guías de centros de hemodiálisis. Nefrología 2006;26 (Supl 8):15-21.

5. Maduell F, Navarro V, García H, Calvo C. Resultados del seguimiento de la dosis de hemodiálisis en tiempo real y en cada sesión. Nefrología 1999; 19:532-7.

6. Molina Núñez M, Gómez J, Álvarez GM, Navarro MJ, Alarcón RM, De Gracia MC, et al. Medida de la dosis de diálisis en una unidad de hemodiálisis. Kt versus Kt/V. Nefrología 2008;28(Supl 4):59.
7. Fernández AV, Soto $S$, Arenas $M$ y cols Estudio comparativo de la dosis de diálisis medida por (Kt) y KtV. 21 Rev Soc Esp Enferm Nefrol 2009; 12(2): 97-102.

8. Maduell F, Vera M, Serra N, Collado S, Carrera M, Fernández $\mathrm{A}$, et al. Kt como control y seguimiento de la dosis en una unidad de hemodiálisis. Nefrología 2008;28:43-7.

9. Lowrie EG, Chertow GM, Lew NL, Lazarews JM, Owen WF. The urea (clearance $x$ dialysis time) product $(\mathrm{Kt})$ as an outcome-based measure of hemodialysis dose. Kidney Int 56. 729-737, 1999.

10. Maduell F, Puchades MJ, Navarro V, Rius A, Torregrosa $E$, Sánchez JJ. Valoración de la medición de la dosis de diálisis con dialisancia iónica en hemodiafiltración on-line. Nefrología 2005; 25:521-6.

11. Teruel JL, Fernández Lucas M, Marcel R, Rodríguez JR, Rivera M, Liaño $F$, et al. Cálculo de la dosis de diálisis mediante dialisancia iónica. Nefrología 2001; 21:78-83.

12. Fernández $A V$, Pereira MS, Vilar MV y cols. Kt como indicador de dosis adecuada en una unidad de hemodiálisis: Estudio prospectivo. Libro de comunicaciones presentadas al XXXIV Congreso Nacional de la SEDEN. Pamplona 2009.

13. Fernández $A V$, Piñero J, Arregui $Y$ y cols. Dializar en tiempos de crisis: comparación de la eficacia dialítica entre una pauta estándar frente a otra optimizando flujo sanguíneo y tiempo efectivo en el paciente en hemodiafiltración on-line. Rev Soc Esp Enferm Nefrol 2012; 15 (1): 33-34.

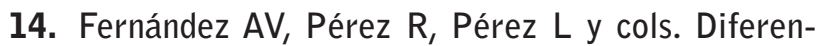
cia entre tiempo programado y tiempo efectivo en hemodiafiltración on-line. ¿Cada minuto cuenta en la eficacia dialítica? Rev Soc Esp Enferm Nefrol 2012; 15 (1): 35-36.

15. Maduell F, Navarro V: Medida y control de la eficacia en hemodiálisis. Diálisis adecuada. Monitorización continua. En Tratado de Hemodiálisis Jofré R,López Gómez JM, Luño J, Pérez García R, Rodriguez Benítez $P$ (eds). Editorial Médica JIMS S.L., Barcelona pgs 243-270, 2006. 
16. Maduell F, García H, Navarro V y Calvo C. Influencia del líquido de diálisis y de la hemodiafiltración sobre la eficacia de la diálisis. Nefrología 16: 347-352. 1996.

17. Vicente JP, Belchí F, Navarro C y cols. Influencia del aumento del flujo del líquido dializante sobre la eficacia de la diálisis. XXVI Congreso de la SEDEN. 2001.

18. Maduell Canals F, Arias Guillén M. Dosis de siálisis. In: Lorenzo-Sellarés V, López-Gómez JM, editors. Nefro- logía al día [Internet]. 2 ed. Barcelona (Spain): Sociedad Española de Nefrología/Plusmedical; 04/12/2012 [cited 2013 Apr 21]. http://dx.doi.org/10.3265/Nefrología.2010.publ.ed80.chapter2811.

19. Angoso M, Alcalde G, Álvarez-Ude F y Arenas MD. Guías SEN de Centros de Hemodiálisis. Gestión de calidad de Hemodiálisis. Nefrología 26 (supl.8): 73-87, 2006. 